\title{
Phase-Field Simulation on the Formation and Collapse Processes of the Rafted Structure in Ni-Based Superalloys
}

\author{
Yuhki Tsukada $^{1, *}$, Yoshinori Murata ${ }^{1}$, Toshiyuki Koyama ${ }^{2}$ and Masahiko Morinaga ${ }^{1}$ \\ ${ }^{1}$ Department of Materials, Physics and Energy Engineering, Graduate School of Engineering, \\ Nagoya University, Nagoya 464-8603, Japan \\ ${ }^{2}$ National Institute for Materials Science, Tsukuba 305-0047, Japan
}

In Ni-based superalloys, the rafted structure is known to form in the early stage of creep and to get into wavy morphology in the final stage of creep at elevated temperatures. This rafting phenomenon is essentially related to the anisotropic relaxation of the lattice misfit between the $\gamma$ and $\gamma^{\prime}$ phases due to the creep strain under the external stress. In this study, in order to simulate comprehensively from the formation to collapse processes of the rafted structure by the phase-field method, a new idea that the anisotropy increases with simulation time is employed in the calculation of the elastic strain energy in alloy. This idea corresponds to the phenomenon that creep strain increases with creep time. The results are in good agreement with the microstructural change observed in practical Ni-based alloys. [doi:10.2320/matertrans.MBW200709]

(Received October 16, 2007; Accepted January 7, 2008; Published February 25, 2008)

Keywords: phase-field model, nickel-based alloys, rafting

\section{Introduction}

Ni-based superalloys consisting of the precipitated $\gamma^{\prime}$ phase ( $\mathrm{L} 1_{2}$ structure) in the $\gamma$ matrix with face-centered cubic structure are applied to gas turbine materials because of their excellent mechanical properties such as creep strength at high temperatures. In aging process, the cuboidal $\gamma^{\prime}$ strengthening phase precipitates and arranges along the $\langle 100\rangle$ crystallographic directions in the $\gamma$ matrix. In creep process, it is known that the rafted structure is formed, i.e. the cuboidal microstructure changes into the lamellar structure, in which plate like $\gamma$ and $\gamma^{\prime}$ phases are stacked alternately, in the early stage of creep. Then, in the final stage of creep life, the rafted structure collapses and gets into wavy morphology. ${ }^{1)}$ The creep strength is strongly related to this morphological change. In fact, there are some reports that the remarkable creep strength originates from the rafted structure, because dislocation movement is interrupted by the $\gamma / \gamma^{\prime}$ interfaces which lay with a large angle against the slip plane. ${ }^{2,3)}$ For this reason, many attentions have been focused on the mechanism of rafting phenomenon.

The study on the rafting mechanism has been performed in elastic and elastic-plastic regimes. ${ }^{4-6)}$ However, it seems not possible to explain all the rafting phenomena in the framework of pure elasticity theory, because there has been some reports that the pre-crept sample forms the rafted structure by the following aging without an external load. ${ }^{7-9)}$ Recently, in the elastic-plastic regime associated with the role of the interfacial dislocations on the $\gamma / \gamma^{\prime}$ interfaces introduced during creep under the external load, Ichitsubo et al. estimated the elastic strain energy and discussed the elastic stability of the rafted structure ${ }^{10)}$ they concluded that the anisotropic relaxation of the lattice mismatch due to the creep dislocations leads to the change in the microstructure from a cuboidal structure into a parallel or normal rafted structure in terms of reduction of the self-strain energy. ${ }^{10)}$ In addition, Ratel et al. reported that the rafted shape and its direction could be explained by the energetic analysis based on the

*Graduate Student, Nagoya University inclusion theory with anisotropic elasticity in the $\gamma$ matrix with plastic strain. ${ }^{11)}$

It is possible to simulate complicated microstructures by the phase-field method using a set of field variables which change continuously in the interfaces. There have been some models of the phase-field method for simulating microstructure evolutions in Ni-Al alloys. ${ }^{12-14)}$ Also, there are a few reports about the simulation of the rafting phenomena, ${ }^{15}$ ) but a comprehensive simulation from the formation to the collapse of the rafted structure has not been reported as long as we know.

The purpose of this study is to perform the comprehensive simulation of the change in the rafted structure corresponding to creep deformation using the phase-field method based on the elastic strain energy calculation taking account of the anisotropic relaxation of the lattice mismatch.

\section{Calculation Model}

In order to simulate morphological change of the $\gamma$ and $\gamma^{\prime}$ phases, the volume fraction of the $\gamma^{\prime}$ phase, $f(\mathbf{r}, t)$, and three long-range order parameters $s_{i}(\mathbf{r}, t)(i=1,2,3)$ which describe the four different domains in the $\mathrm{L}_{2}$ phase structure are chosen as field variables. These field variables vary spatially (r) and temporally $(t)$. Usually, alloy composition, $c(\mathbf{r}, t)$, is used as a field variable ${ }^{12-15)}$ but in this study, $f(\mathbf{r}, t)$ is used instead of $c(\mathbf{r}, t)$, because $f(\mathbf{r}, t)$ is suitable to treat the multi-component system when the phase field method is applied to the practical Ni-based alloys. The temporal evolution of the field variables is given by solving the following Cahn-Hilliard and Ginzburg-Landau equations:

$$
\begin{aligned}
\frac{\partial f(\mathbf{r}, t)}{\partial t} & =M \nabla^{2} \frac{\delta G_{\mathrm{sys}}}{\delta f(\mathbf{r}, t)}, \\
\frac{\partial s_{i}(\mathbf{r}, t)}{\partial t} & =-L \frac{\delta G_{\mathrm{sys}}}{\delta s_{i}(\mathbf{r}, t)} \quad(i=1,2,3),
\end{aligned}
$$

where $G_{\text {sys }}$ is the total free energy of the system, $M$ is the diffusion mobility and $L$ is the structural relaxation coefficient. The total free energy of the system is given by the sum of the chemical free energy $\left(G_{c}\right)$, interfacial energy 


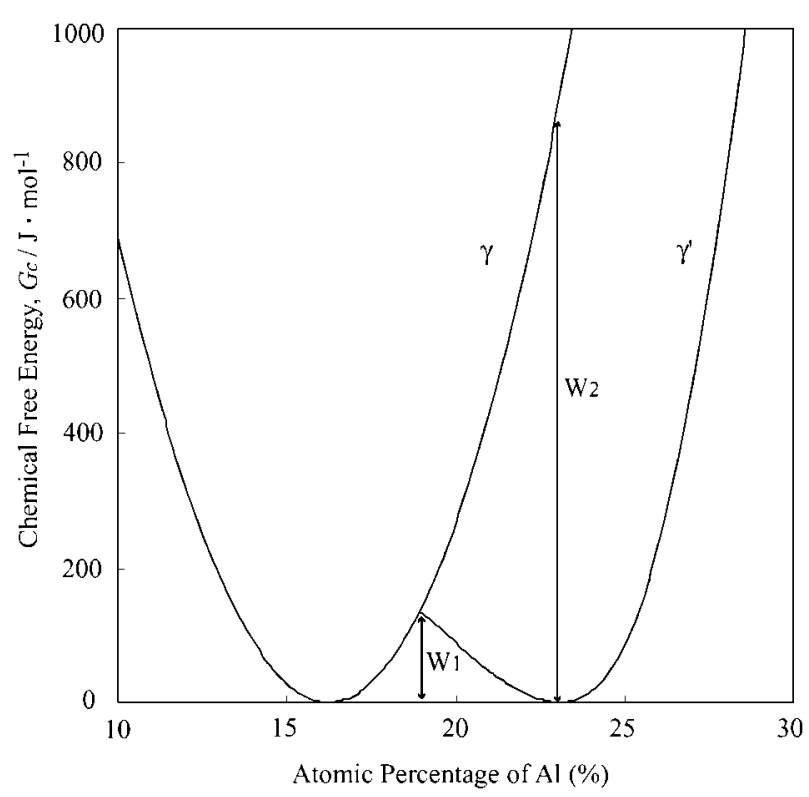

Fig. 1 Calculated Gibbs energy curve of the $\left(\gamma+\gamma^{\prime}\right)$ two-phase region in $\mathrm{Ni}-\mathrm{Al}$ alloy at $1273 \mathrm{~K}$.

(gradient terms) $\left(E_{\text {surf }}\right)$ and elastic strain energy $\left(E_{\text {str }}\right)$, and it is written as

$$
G_{\text {sys }}=\int_{\mathbf{r}}\left[G_{c}\left(f, s_{i}\right)+E_{\text {surf }}\left(f, s_{i}\right)+E_{\text {str }}\left(f, s_{i}\right)\right] d \mathbf{r} .
$$

In this study, the Gibbs free energy curve of $\left(\gamma+\gamma^{\prime}\right)$ twophase region is expressed by the chemical free energy function proposed by Koyama. ${ }^{16)}$ The function is

$$
G_{c}=16 W_{1} f^{2}(1-f)^{2}+W_{2}\left(f-s_{1} s_{2} s_{3}\right)^{2},
$$

where $W_{1}$ and $W_{2}$ are the coefficients determined by the Gibbs energy calculation based on the sub-lattice model using the thermodynamic database. The first term in eq. (4) is the double-well function which assures the separation of $\gamma$ and $\gamma^{\prime}$ phases. The second term assures the ordering of the $\gamma^{\prime}$ phase and the local minima of $G_{c}$ appear at $\left(s_{1}, s_{2}, s_{3}\right)=$ $(1,1,1),(1,-1,-1),(-1,1,-1)$ and $(-1,-1,1)$, because $f=1$ where the $\gamma^{\prime}$ phase exists. ${ }^{12)}$ These four cases correspond to the four different domains in the $\mathrm{L}_{2}$ structure. Figure 1 shows the calculated Gibbs energy curve of $\left(\gamma+\gamma^{\prime}\right)$ two-phase region in $\mathrm{Ni}-\mathrm{Al}$ alloy at $1273 \mathrm{~K}$ based on the twosub-lattice model. The values of $W_{1}$ and $W_{2}$ can be obtained as the values shown in Fig. 1. In a multi-component system, Gibbs energy curve of the $\left(\gamma+\gamma^{\prime}\right)$ two-phase region gives $W_{1}$ and $W_{2}$ values in a similar manner used in $\mathrm{Ni}-\mathrm{Al}$ system.

In this study, the interfacial energy is employed as the gradient energy of the field variables, that is

$$
E_{\text {surf }}=\kappa_{f}(\nabla f)^{2}+\frac{1}{2} \kappa_{s}\left\{\left|\nabla s_{1}\right|^{2}+\left|\nabla s_{2}\right|^{2}+\left|\nabla s_{3}\right|^{2}\right\},
$$

where $\kappa_{f}$ and $\kappa_{s}$ are the gradient energy coefficients. ${ }^{17)}$ Since accurate data on experimental interfacial energy is not available even in Ni-Al binary alloy, the gradient energy coefficients are determined so that the calculation results can reproduce the microstructure of the practical $\mathrm{Ni}-\mathrm{Al}$ alloys.

The elastic strain energy arising from the lattice misfit between the $\gamma$ and $\gamma^{\prime}$ phases is estimated based on the micromechanics. ${ }^{18,19)}$ The elastic strain is expressed as

$$
\varepsilon_{i j}^{e l}(\mathbf{r}, t)=\varepsilon_{i j}^{c}(\mathbf{r}, t)-\varepsilon_{i j}^{0}(\mathbf{r}, t),
$$

where $\varepsilon_{i j}^{c}$ and $\varepsilon_{i j}^{0}$ represent the constrained strain and eigenstrain, respectively. Here, the eigenstrain is regarded as the liner function of $f(\mathbf{r}, t)$ and expressed as

$$
\varepsilon_{i j}^{0}(\mathbf{r}, t)=\varepsilon_{i j}^{*}\left\{f(\mathbf{r}, t)-f_{0}\right\},
$$

where $\varepsilon_{i j}^{*}$ represents the total misfit strain (eigenstrain) and $f_{0}$ is the average volume fraction of the $\gamma^{\prime}$ phase. In the elastic equilibrium condition, the elastic strain is written as

$$
\begin{aligned}
& \varepsilon_{k l}^{c}(\mathbf{r}, t)-\varepsilon_{k l}^{0}(\mathbf{r}, t) \\
& \quad=\int_{\mathbf{k}}\left\{\varepsilon_{k l}^{c}(\mathbf{k}, t)-\varepsilon_{k l}^{0}(\mathbf{k}, t)\right\} \exp (i \mathbf{k} \cdot \mathbf{r}) \frac{d \mathbf{k}}{(2 \pi)^{3}},
\end{aligned}
$$

where

$$
\varepsilon_{k l}^{c}(\mathbf{k}, t)=\frac{1}{2} C_{p q m n}\left\{k_{k} k_{q} \Omega_{p l}(\mathbf{k})+k_{l} k_{q} \Omega_{p k}(\mathbf{k})\right\} \varepsilon_{m n}^{0}(\mathbf{k}, t) .
$$

Here, $C_{i j k l}$ is the elastic-constant tensor and is assumed to be elastically anisotropic. $k$ is the unit vector of the reciprocalspace vector $\mathbf{k}, \varepsilon_{k l}^{c}(\mathbf{k}, t)$ and $\varepsilon_{k l}^{0}(\mathbf{k}, t)$ are the Fourier transformation of $\varepsilon_{k l}^{c}(\mathbf{r}, t)$ and $\varepsilon_{k l}^{0}(\mathbf{r}, t)$, respectively, and $\Omega_{i k}^{-1}(\mathbf{k})$ is given by $\Omega_{i k}^{-1}(\mathbf{k})=C_{i j k l} k_{j} k_{l}$. The elastic strain energy is then given by

$$
E_{\mathrm{str}}=\frac{1}{2} C_{i j k l}\left\{\varepsilon_{i j}^{c}(\mathbf{r}, t)-\varepsilon_{i j}^{0}(\mathbf{r}, t)\right\}\left\{\varepsilon_{k l}^{c}(\mathbf{r}, t)-\varepsilon_{k l}^{0}(\mathbf{r}, t)\right\} .
$$

The misfit strain between $\gamma$ and $\gamma^{\prime}$ phases is given by

$$
\varepsilon^{T}=\left(\begin{array}{ccc}
\varepsilon_{0} & 0 & 0 \\
0 & \varepsilon_{0} & 0 \\
0 & 0 & \varepsilon_{0}
\end{array}\right),
$$

and

$$
\varepsilon_{0}=\frac{a_{\gamma^{\prime}}-a_{\gamma}}{a_{\gamma}}
$$

Here, $a_{\gamma}$ and $a_{\gamma^{\prime}}$ are the lattice parameters of the $\gamma$ and $\gamma^{\prime}$ phases, respectively.

In this study, the plastic deformation observed in creep experiments are introduced into the matrix with the misfit strain by $\varepsilon_{p}$ along the [001] tensile stress. ${ }^{11)}$ In this case, the lateral directions undergo a plastic strain of $-\varepsilon_{p} / 2$ and hence the misfit strain of the the $\gamma^{\prime}$ phase induced by the creep deformation is expressed as

$$
\varepsilon^{P}=\left(\begin{array}{ccc}
\varepsilon_{P} / 2 & 0 & 0 \\
0 & \varepsilon_{P} / 2 & 0 \\
0 & 0 & -\varepsilon_{P}
\end{array}\right) .
$$

As a result, when the misfit strain and the creep deformation-induced misfit strain (creep strain) exist, the total misfit strain (eigenstrain) $\varepsilon^{*}$ is expressed as the sum of $\varepsilon^{T}$ and $\varepsilon^{P}$, that is

$$
\varepsilon^{*}=\varepsilon^{T}+\varepsilon^{P}
$$

\section{Results and Discussion}

\subsection{Elastic strain energy}

The elastic constant of the $\gamma$ phase is different from that of the $\gamma^{\prime}$ phase, but elastic strain energy caused by the difference 


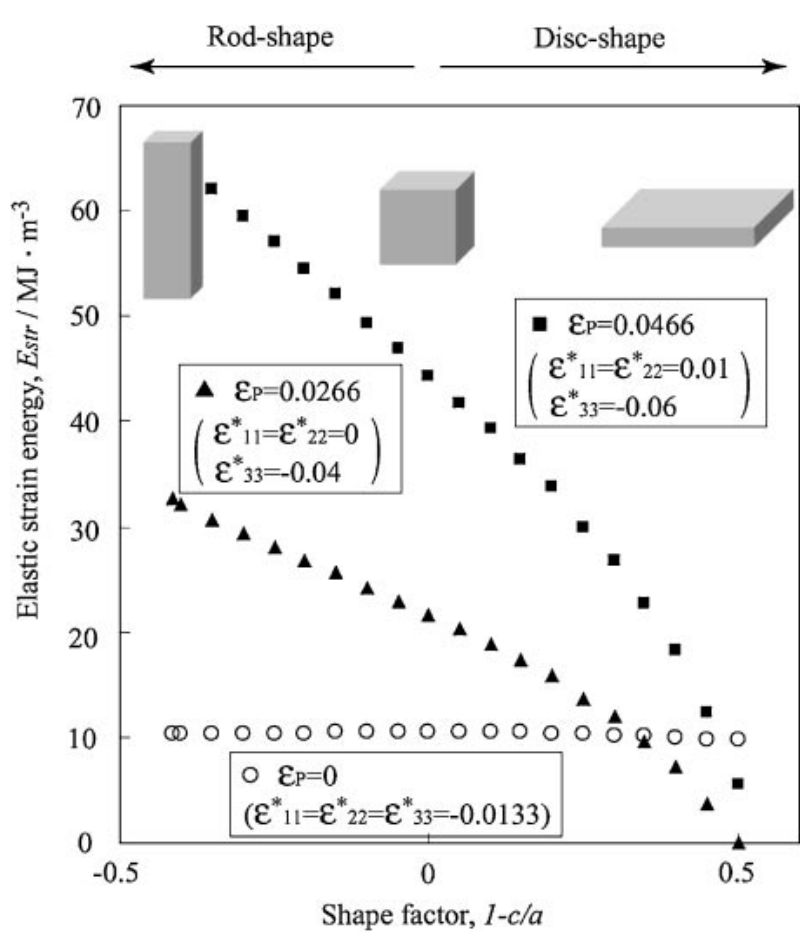

Fig. 2 Change in elastic strain energy with the shape change of the $\gamma^{\prime}$ phase as a function of $1-c / a$ as the eigenstrain changes from cubic symmetry into tetragonal symmetry. The shape of the $\gamma^{\prime}$ phase is represented by $a \times a \times c$ in dimensions.

in the elastic constant is negligible when the creep strain exists. In this study, therefore, the same elastic constants as pure $\mathrm{Ni}$, which are $c_{11}=250.8 \mathrm{GPa}, c_{12}=150.0 \mathrm{GPa}$ and $c_{44}=123.5 \mathrm{GPa},{ }^{20)}$ were used for the two phases in the calculation of the elastic strain energy. Figure 2 shows change in the elastic strain energy with the shape change of the $\gamma^{\prime}$ phase as a function of $1-c / a$, where the shape of the $\gamma^{\prime}$ phase is represented by $a \times a \times c$ in dimensions. In this calculation, $\varepsilon_{0}=-0.0133$ was used as the misfit strain and the volume fraction of the $\gamma^{\prime}$ phase was set to be 0.5 . In Fig. 2, the calculation results are shown in three cases, i.e., the creep strain $\varepsilon_{P}$ of the $\gamma$ phase along the [001] direction are $0(\bigcirc), 0.0266(\boldsymbol{\Delta})$ and $0.0466(\boldsymbol{\square})$, respectively. Here, the axial ratio $c / a$ of the rod-shape and the disc-shape of the $\gamma^{\prime}$ phases are assumed to be 1.414 and 0.5 , respectively, when the volume fraction of the $\gamma^{\prime}$ phase was set to be 0.5 . Generally, the elastic strain energy increases when the creep strain is introduced, and hence each of the three cases in Fig. 2 cannot be compared with each other. In other words, each case predicts the stable shape of the $\gamma^{\prime}$ phase at a creep strain. When $\varepsilon_{P}=0$, the eigenstrain has spherical symmetry and the value of the elastic strain energy is independent of the shape change of the $\gamma^{\prime}$ phase. However, as the creep strain $\varepsilon_{P}$ increases, the spherical symmetry of the eigenstrain changes into tetragonal symmetry. As a result, the elastic strain energy decreases with rafting, though it increases for the rodshape structure; see the case of $\varepsilon_{P}=0.0266$ in Fig. 2. Thus, the anisotropic relaxation of the lattice mismatch due to the creep strain reduces the elastic strain energy, resulting in the change in the shape of the $\gamma^{\prime}$ phase from the cuboidal structure to the rafted structure. Here, if the creep deformation proceeds until $\varepsilon_{11}^{*}\left(=\varepsilon_{22}^{*}\right)$ becomes a positive value, i.e. if

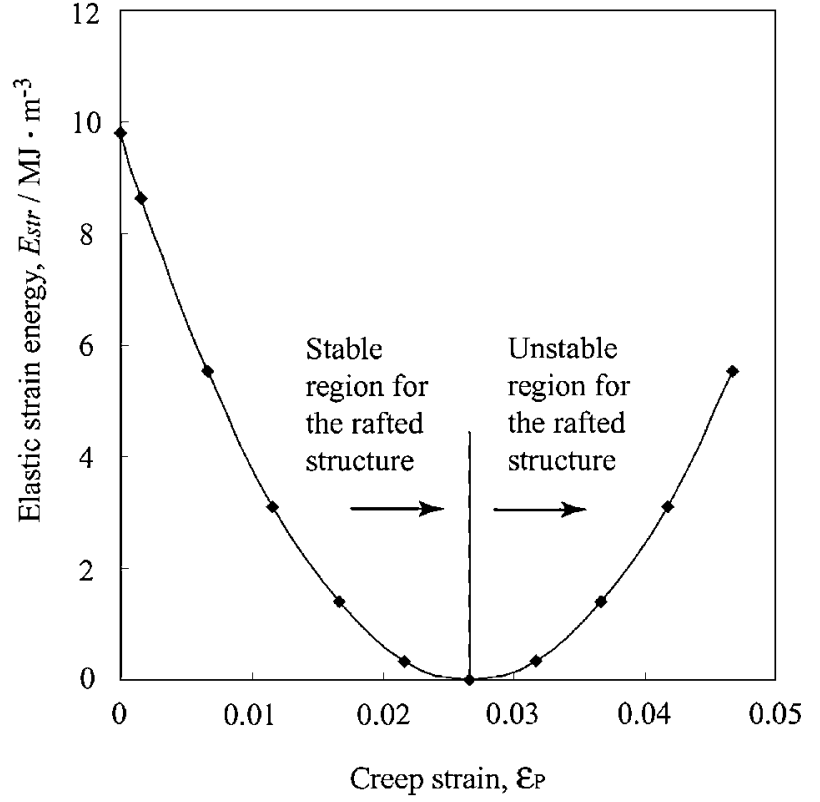

Fig. 3 Change in elastic strain energy with increasing the creep strain $\varepsilon_{P}$ at the shape factor, $1-c / a=0.5$. The misfit strain is set to be $\varepsilon_{0}=$ -0.0133 .

$\varepsilon_{P}$ exceeds 0.0266 in this case, a remarkable result is obtained concerning the stable shape of the $\gamma^{\prime}$ phase. Figure 3 shows the change in the elastic strain energy at $1-c / a=0.5$ with increasing the creep strain gradually. From the figure, it can be said that the rafted structure becomes stable as the creep strain increases, but it becomes unstable when $\varepsilon_{P}$ exceeds 0.0266 , i.e. when $\varepsilon_{11}^{*}\left(=\varepsilon_{22}^{*}\right)$ becomes a positive value. Figure 4 shows the relation between the stable shape of the $\gamma^{\prime}$ phase and the ratio of $t_{1}=\varepsilon_{11}^{*} / \varepsilon_{33}^{*}{ }^{18)}$ When the creep strain exists and $t_{1}$ is between 0 and 1 , the (001) raft is the stable structure and the rafting phenomena occurs. However, if the creep strain increases and $t_{1}$ becomes less than 0 , i.e. if $\varepsilon_{11}^{*}\left(=\varepsilon_{22}^{*}\right)$ becomes a positive value $\left(\varepsilon_{11}^{*} / \varepsilon_{33}^{*}<0\right)$, the stable orientation of the raft plane tilts, which leads to the destabilization of the rafted structure.

Based on the analysis of the elastic strain energy as shown in Figs. 2, 3 and 4, the formation and collapse processes of the rafted structure can be understood. Actually there has been a report that the stable orientation of the lamellar plane changes as the creep deformation proceeds. ${ }^{21)}$

\subsection{Phase-field simulation}

Simulations were performed by solving the two sets of equations, eqs. (1) and (2) numerically, using the explicit method under the periodic boundary conditions. The coefficients of the chemical free energy in eq. (4) were determined as $W_{1}=133$ and $W_{2}=877 \mathrm{~J} / \mathrm{mol}$ from the Gibbs energy curve at $1273 \mathrm{~K}$ calculated based on the thermodynamic database ${ }^{22)}$ as shown in Fig. 1. The average volume fraction of the $\gamma^{\prime}$ phase was set to be $f_{0}=0.5$ and the gradient energy coefficients were chosen as $\kappa_{f}=\kappa_{s}=$ $5.0 \times 10^{-15} \mathrm{Jm}^{2} / \mathrm{mol}$. In the calculation of the elastic strain energy, values $c_{11}=250.8 \mathrm{GPa}, c_{12}=150.0 \mathrm{GPa}, c_{44}=$ $123.5 \mathrm{GPa}^{20)}$ and $\varepsilon_{0}=-0.0133$ were used in this study. Time step $\Delta t$ was selected to be 0.2 so as to maintain the stable solution. 


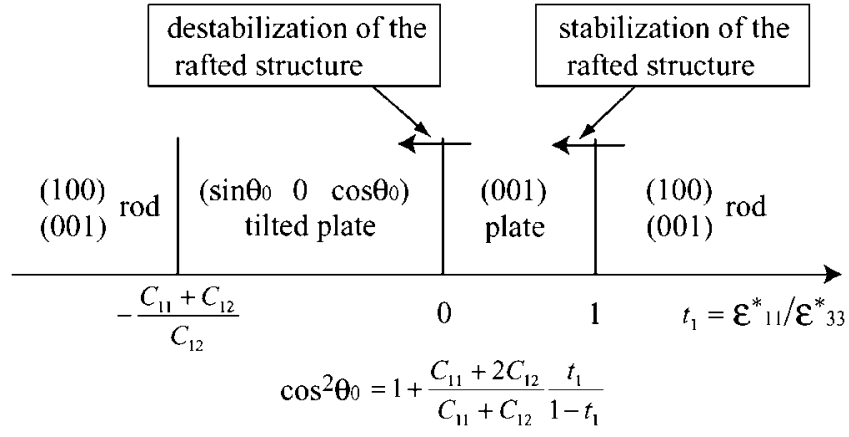

Fig. 4 Schematic interpretation of the relation between the stable shape of the $\gamma^{\prime}$ phase and the ratio of $\varepsilon_{11}^{*}$ to $\varepsilon_{33}^{*}$.

Figure 5 shows the 2D morphological evolution of the $\gamma^{\prime}$ phase for $f_{0}=0.5$ at $1273 \mathrm{~K}$. The $\gamma^{\prime}$ phase is expressed as white area. First of all, simulation was carried out using only the misfit strain without any creep strain up to $t=5000$, assuming that the specimen was aged simply at this temperature. The result is shown in Fig. 5(a). In this case, the eigenstrain has spherical symmetry, and the cuboidal shape of the $\gamma^{\prime}$ phase arranged along the $\langle 100\rangle$ crystallographic directions is observed due to only the anisotropic elastic interaction. After $t=5000$, the increase of the creep strain at the constant rate $d \varepsilon_{P} / d t=6.66 \times 10^{-7}$ under the [001] tensile stress is introduced by assuming that the creep strain increases during creep. This creep strain relaxes the lattice mismatch in (001) plane. Thus, during $t=5000-45000$ (Fig. 5(a)-(e)), it is observed that the cuboidal $\gamma^{\prime}$ structure gradually changes into the rafted structure and the rafted structure becomes stable in terms of the elastic strain energy. When $t$ becomes more than 45000 , i.e. when $\varepsilon_{11}^{*}\left(=\varepsilon_{22}^{*}\right)$ becomes a positive value, the rafted structure collapses and gets into wavy morphology as shown in Fig. 5(e)-(h). This means that the rafted structure becomes unstable. This is consistent with the fact that the rafted structure is stable when $\varepsilon_{11}^{*} / \varepsilon_{33}^{*}$ is between 0 and 1 , and the rafted structure is unstable when $\varepsilon_{11}^{*} / \varepsilon_{33}^{*}$ is less than 0 , as mentioned in Section 3.1.
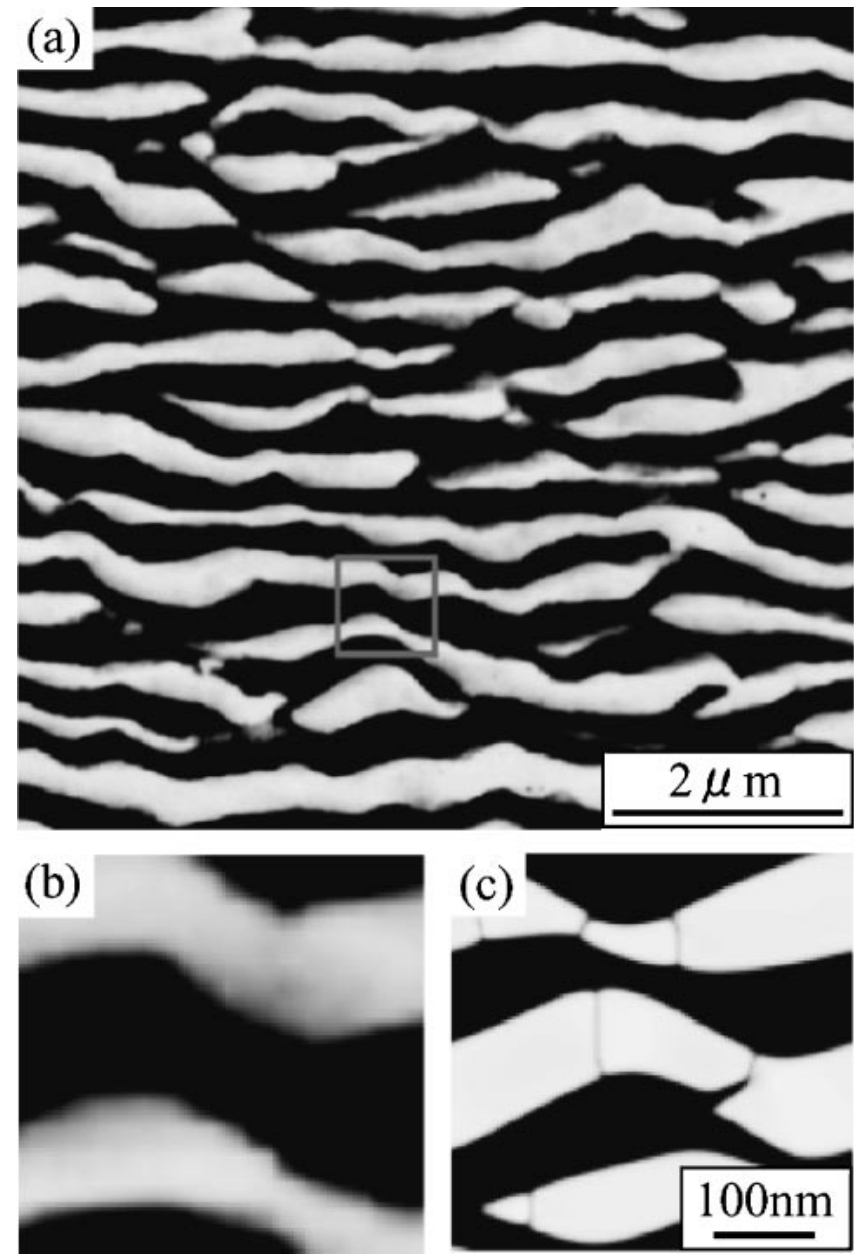

Fig. 6 Microstructure of a nickel based superalloy crept at $1193 \mathrm{~K}$ for $1145 \mathrm{~h}$ and the result of phase-field simulation in this work. (a) Microstructure of a superalloy, (b) Enlarged figure of a part in (a), and (c) A phase-field simulation result at the reduced simulation time $\mathrm{t}=75000$.
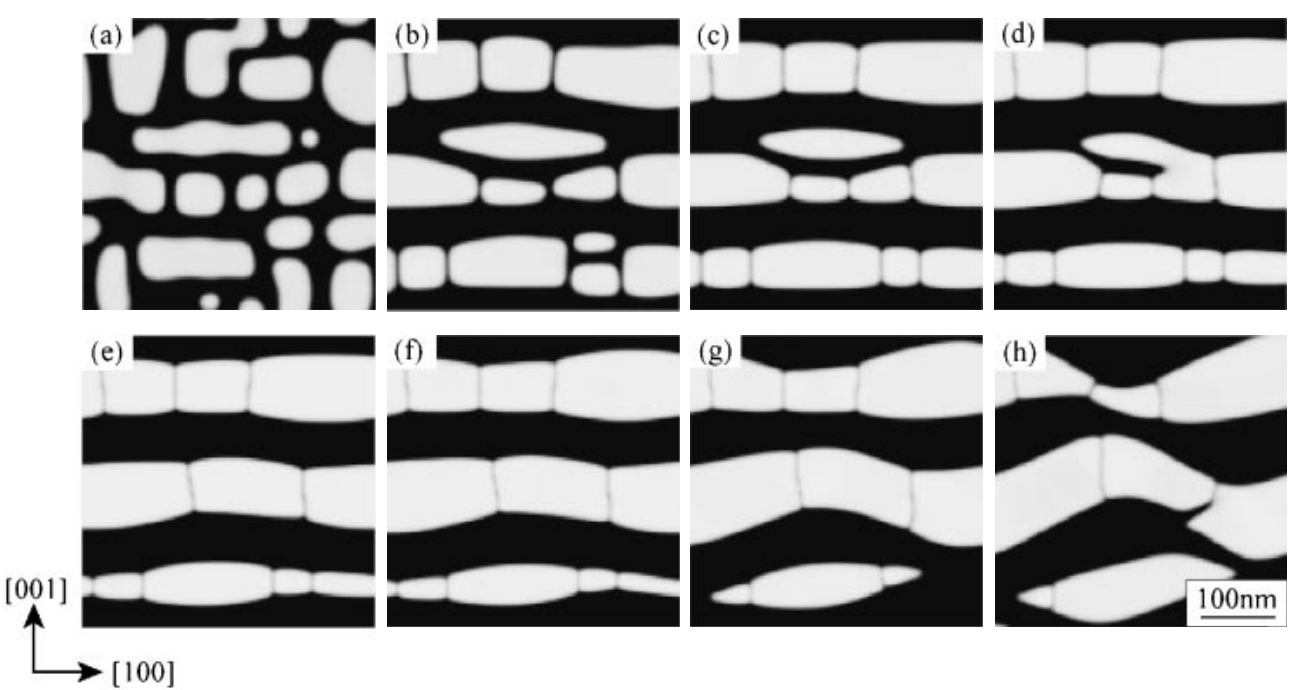

Fig. 5 Results on two-dimensional phase-field simulation showing the change of the $\gamma^{\prime}$ phase for $f_{0}=0.5$ at $1273 \mathrm{~K}$. (a) $\mathrm{t}=5000$, (b) $\mathrm{t}=15000$, (c) $\mathrm{t}=25000$, (d) $\mathrm{t}=35000$, (e) $\mathrm{t}=45000$, (f) $\mathrm{t}=55000$, (g) $\mathrm{t}=65000$ and (h) $\mathrm{t}=75000$. Time expressed here are reduced simulation time. 
Figure 6(a) shows a microstructure obtained from the experiment of a nickel based superalloy (Ni-14.0 mol\%Cr$10.8 \% \mathrm{Al}-3.8 \% \mathrm{Co}-2.5 \% \mathrm{~W}-1.9 \% \mathrm{Ta}-1.5 \% \mathrm{Ti}-0.3 \% \mathrm{Mo}){ }^{1)}$ which was designed for land-base gas-turbines, crept at $1193 \mathrm{~K}$ for $1145 \mathrm{~h}$. A part marked by a square in Fig. 6(a) is enlarged in Fig. 6(b). Figure 6(c) shows the simulation microstructure at $t=75000$ obtained in this study. The simulation result is in good agreement with the microstructural change of practical Ni-based alloys.

\section{Conclusions}

The phase-field method has been adopted to simulate comprehensively from the formation to collapse process of the rafted structure in Ni-based alloys. In this work, on the basis of the elastic-plastic consideration, anisotropic relaxation of the lattice misfit between the $\gamma$ and $\gamma^{\prime}$ phases due to the creep strain is introduced by increasing the creep strain $\varepsilon_{P}$ with simulation time in a constant rate. The microstructural evolution of the $\gamma^{\prime}$ phase in the creep process is reproduced well in this simulation; the stabilization and the following destabilization of the rafted structure. This result is in good agreement with the microstructural change of practical Nibased superalloys.

\section{REFERENCES}

1) Y. Murata, R. Hashizume, A. Yoshinari, N. Aoki, M. Morinaga and Y. Fukui: Superalloys 2000, (The Minerals, Metal \& Materials Society, 2000) pp. 285-294.
2) D. D. Pearson, F. D. Lemkey and B. H. Kear: Superalloys 1980, (The Minerals, Metal \& Materials Society, 1980) pp. 513-520.

3) J. K. Tien and R. P. Gamble: Metall. Trans. 3 (1972) 2157-2162.

4) J. K. Tien and S. M. Copley: Metall. Trans. 2 (1971) 215-220.

5) F. R. N. Nabarro, C. M. Cress and P. Kotschy: Acta Mater. 44 (1996) 3189-3198.

6) S. Socrate and D. M. Parks: Acta Metall. Mater. 41 (1993) 2185-2209.

7) N. Matan, D. C. Cox, C. M. F. Rae and R. C. Reed: Acta Mater. 47 (1999) 2031-2045.

8) M. Véron, Y. Bréchet and F. Louchet: Scr. Mater. 34 (1996) 18831886.

9) M. Véron and P. Bastie: Acta Mater. 45 (1997) 3277-3282.

10) T. Ichitsubo, D. Koumoto, M. Hirao, K. Tanaka, M. Osawa, T. Yokokawa and H. Harada: Acta Mater. 51 (2003) 4033-4044.

11) N. Ratel, G. Bruno, P. Bastie and T. Mori: Acta Mater. 54 (2006) 50875093.

12) V. Vaithyanathan and L. Q. Chen: Acta Mater. 50 (2002) 4061-4073.

13) J. Z. Zhu, T. Wang, S. H. Zhou, Z. K. Liu and L. Q. Chen: Acta Mater. 52 (2004) 833-840.

14) J. Z. Zhu, T. Wang, A. J. Ardell, S. H. Zhou, Z. K. Liu and L. Q. Chen: Acta Mater. 52 (2004) 2837-2845.

15) M. P. Gururajan and T. A. Abinandanan: Acta Mater. 55 (2007) 50155026.

16) T. Koyama: The 19th Computational Mechanics Conference, (The Japan Society of Mechanical Engineers, 2006) pp. 357-358.

17) J. W. Cahn and J. E. Hilliard: J. Chem. Phys. 28 (1958) 258-267.

18) A. G. Khachaturyan: Theory of structural transformation in solids, (Wiley, New York, 1983).

19) T. Mura: Micromechanics of Defects in Solids, 2nd revised ed., (Kluwer Academic, 1991).

20) Kinzoku data book, 2nd revised ed., (The Japan Inst. Metals, 1984).

21) K. Tanaka, T. Ichitsubo, K. Kishida and H. Inui: Report of the 123rd Committee on Heat-Resisting Metal and Alloys, (Japan Society of the Promotion of Science, 2005) pp. 147-152.

22) I. Ansara, N. Dupin, H. L. Lukas and B. Sundman: J. Alloy. Compd. 247 (1997) 20-30. 